\title{
Weed Management in White Beans with Soil-Applied Grass Herbicides plus Halosulfuron
}

\author{
Nader Soltani*, Christy Shropshire, Peter H. Sikkema \\ University of Guelph Ridgetown Campus, Ridgetown, Ontario, Canada \\ Email: *soltanin@uoguelph.ca
}

How to cite this paper: Soltani, N., Shropshire, C. and Sikkema, P.H. (2020) Weed Management in White Beans with SoilApplied Grass Herbicides plus Halosulfuron. American Journal of Plant Sciences, 11, 1998-2011.

https://doi.org/10.4236/ajps.2020.1112141

Received: October 22, 2020

Accepted: December 11, 2020

Published: December 14, 2020

Copyright $\odot 2020$ by author(s) and Scientific Research Publishing Inc. This work is licensed under the Creative Commons Attribution International License (CC BY 4.0).

http://creativecommons.org/licenses/by/4.0/

\begin{abstract}
There are a limited number of soil-applied herbicides available for broad-spectrum weed control in dry bean production in Ontario, Canada. Four experiments were conducted from 2017 to 2019 in southwestern Ontario to compare the efficacy of six soil-applied grass herbicides [trifluralin (600 $\mathrm{g}$ ai $\left.\mathrm{ha}^{-1}\right)$, ethalfluralin $\left(810 \mathrm{~g}\right.$ ai ha $\left.\mathrm{h}^{-1}\right)$, pendimethalin (1080 $\mathrm{g}$ ai ha $\left.\mathrm{ha}^{-1}\right)$, S-metolachlor (1050 $\mathrm{g}$ ai ha $\left.{ }^{-1}\right)$, dimethenamid-p (544 $\mathrm{g}$ ai ha $\left.{ }^{-1}\right)$ and EPTC $\left(3400 \mathrm{~g}\right.$ ai ha $\left.\mathrm{h}^{-1}\right)$ ] and halosulfuron $\left(35 \mathrm{~g}\right.$ ai ha $\left.{ }^{-1}\right)$ applied alone and in combination, applied preplant incorporated (PPI), on white bean tolerance and yield, and weed control efficacy. There was no white bean injury from the herbicide treatments evaluated. Grass herbicides (trifluralin, ethalfluralin, pendimethalin, S-metolachlor dimethenamid-P and EPTC) controlled velvetleaf $0 \%$ - 82\%, pigweeds $87 \%$ - 99\%, common ragweed 0\% - 93\%, common lambsquarters $81 \%$ - $99 \%$, wild mustard $0 \%-71 \%$, barnyardgrass $98 \%-100 \%$ and green foxtail $98 \%$ - 99\%. Halosulfuron controlled velvetleaf $98 \%$, pigweeds $94 \%$, common ragweed $90 \%-94 \%$, common lambsquarters $97 \%$, wild mustard $98 \%-100 \%$, barnyardgrass $19 \%-24 \%$ and green foxtail $20 \%-25 \%$. Tankmixes of halosulfuron with soil-applied grass herbicides provided $\geq 93 \%$ control of the weed species evaluated. Reduction in density and biomass generally followed the same trend as visible control with herbicide treatments evaluated. Weed interference reduced white bean seed yield $70 \%$. Seed yield was $53 \%-66 \%$ of the weed-free control with trifluralin, ethalfluralin, pendimethalin, S-metolachlor and dimethenamid-P, $81 \%$ of the weed-free control with EPTC, $58 \%$ of the weed-free control with halosulfuron, and $87 \%-95 \%$ of the weed-free control with halosulfuron tankmixes with the grass herbicides evaluated. Based on these results, halosulfuron in combination with trifluralin, ethalfluralin, pendimethalin, S-metolachlor, dimethenamid-p and EPTC, applied PPI at rates evaluated, can be used to effectively control common annual grass and broadleaf weeds in white beans.
\end{abstract}




\section{Keywords}

Weed Density, Weed Biomass, Seed Moisture, Seed Yield, Visible Control, Phaseolus vulgaris

\section{Introduction}

Dry bean (Phaseolus vulgaris L.) is an important field crop grown in southwestern Ontario that fits well in a typical Ontario crop rotation of corn, soybean and wheat. Dry bean growers produced over 120,000 tonnes of dry beans on nearly 52,000 hectares with a farm gate value of nearly $\$ 115,000,000$ in 2017 [1]. Weeds can interfere with dry bean growth and development and cause substantial losses in seed yield and quality if not adequately controlled [2] [3] [4]. White navy bean is the most commonly grown market class of dry beans in Ontario. A limited number of soil-applied herbicides are available for weed control in white bean production in Ontario. Currently, only two soil-applied herbicides are available for broadleaf weed control in white bean production in Ontario, imazethapyr and halosulfuron [5]. Although imazethapyr is a very efficacious soil-applied broadleaf herbicide, it has a narrow margin of crop safety, especially in the small-seeded market classes of beans, specifically white and black beans. New research is needed to identify new soil-applied herbicides/tankmixes for efficacious broad-spectrum weed control in white beans.

Halosulfuron is a Group 2 soil and foliar applied sulfonylurea herbicide that was recently registered at 25 to $50 \mathrm{~g}$ ai ha ${ }^{-1}$ for use in dry beans and has become a popular herbicide option for the control of common annual broadleaf weeds in Ontario [5]. Halosulfuron inhibits the acetolactate synthase (ALS) enzyme which is needed for synthesis of branched-chain amino acids including isoleucine, leucine and valine [6]. Halosulfuron controls common annual broadleaf weeds in Ontario such as redroot pigweed (Amaranthus retroflexus L.), common lambsquarters (Chenopodium album L.), common ragweed (Ambrosia artemisiifolia L.), wild mustard (Sinapis arvensis L.) and velvetleaf (Abutilon theophrasti Medic.). However, halosulfuron does not adequately control grasses and needs to be tankmixed with a selective grass herbicide to provide broad-spectrum control of common annual weeds in dry bean production in Ontario [7] [8] [9].

Grass herbicides available in Ontario that have the potential to be used with halosulfuron include trifluralin, ethalfluralin, pendimethalin, S-metolachlor, dimethenamid-P and EPTC. Trifluralin is a Group 3 dinitroaniline herbicide that controls most grasses and some annual broadleaf weeds such as common lambsquarters and pigweeds [5]. Ethalfluralin is another Group 3 dinitroanaline herbicide that controls most grasses and some broadleaf weeds such as pigweeds, common lambsquarters, ladysthumb, Russian thistle (Salsola tragus L.), kochia (Brassia scoparia subsp. densiflora) and wild buckwheat (Polygonum convolvulus L.) [5] [10]. Ethalfluralin is not currently registered for use in white bean or 
any other market class of dry beans in Ontario [5]. Pendimethalin is a Group 3 dinitroaniline herbicide that controls grasses such as barnyardgrass [Echinochloa crus-galli (L.) Beauv.], foxtail species (Setaria spp.), crabgrass species (Digitaria spp.), fall panicum (Panicum dichotomiflorum Michx.) and broadleaf weeds such as common lambsquarters and pigweed species [5]. S-metolachlor is a Group 15 chloroacetanilide herbicide that control crabgrass, witchgrass (Panicum capillare L.), barnyardgrass, foxtails, nightshades (Solanum spp.), pigweeds and waterhemp [5]. Dimethenamid-P is another Group 15 chloroacetamide grass herbicide that controls barnyardgrass, foxtails, crabgrass and broadleaf weeds such as pigweeds, nightshades, and waterhemp (Amaranthus rudis Sauer) [5] [10]. EPTC is a Group 15 thiocarbamate herbicide that controls annual grasses such as barnyardgrass, foxtails, fall panicum, and wild oats (Avena fatua L.), plus yellow nutsedge (Cyperus esculentus) and some annual broadleaf weeds such as common ragweed, pigweed species, chickweed (Stellaria media L.) and nightshades [5] [10].

It is critical to determine the appropriate partner grass herbicide for halosulfuron based on weed species composition in each individual field for further adoption of halosulfuron in white bean production in Ontario. To our knowledge, no study has cumulatively compared the tolerance and efficacy of trifluralin, ethalfluralin, pendimethalin, S-metolachlor, dimethenamid-P, EPTC, halosulfuron, trifluralin + halosulfuron, ethalfluralin + halosulfuron, pendimethalin + halosulfuron, S-metolachlor + halosulfuron, dimethenamid-P + halosulfuron, and EPTC + halosulfuron, applied preplant incorporated (PPI), for broad-spectrum weed control in white beans.

The purpose of this study was to compare the efficacy of six soil-applied grass herbicides [trifluralin ( $600 \mathrm{~g}$ ai ha $\left.{ }^{-1}\right)$, ethalfluralin $\left(810 \mathrm{~g}\right.$ ai ha $\left.{ }^{-1}\right)$, pendimethalin (1080 $\left.\mathrm{g}_{\text {ai ha }} \mathrm{h}^{-1}\right)$, S-metolachlor (1050 $\mathrm{g}$ ai ha $\left.{ }^{-1}\right)$, dimethenamid-P (544 $\mathrm{g}$ ai ha ${ }^{-1}$ ) and EPTC (3400 $\mathrm{g}$ ai ha $\left.\mathrm{h}^{-1}\right)$ ] and halosulfuron (35 $\mathrm{g}$ ai ha $\mathrm{ha}^{-1}$ ) applied alone and in combination, applied PPI, on white bean tolerance and yield, and weed control efficacy.

\section{Materials and Methods}

Field experiments (total of 4) were conducted in 2018 and 2019 at the University of Guelph Ridgetown Campus, Ridgetown, Ontario, Canada and in 2017 and 2019 at the Huron Research Station, Exeter, Ontario, Canada. Seedbed preparation at all sites consisted of fall moldboard plowing followed by two passes with a field cultivator with rolling basket harrows in the spring.

The experiment was arranged in a randomized block design with treatments replicated four times. Treatments included a weedy and weed-free control and trifluralin (600 $\mathrm{g}$ ai ha $\left.{ }^{-1}\right)$, ethalfluralin $\left(810 \mathrm{~g}\right.$ ai ha $\left.\mathrm{ha}^{-1}\right)$, pendimethalin (1080 $\mathrm{g}$ ai $\mathrm{ha}^{-1}$ ), S-metolachlor (1050 $\mathrm{g}$ ai ha ${ }^{-1}$ ), dimethenamid-P (544 $\mathrm{g}$ ai ha ${ }^{-1}$ ), EPTC $\left(3400 \mathrm{~g}\right.$ ai ha $\left.{ }^{-1}\right)$, halosulfuron (35 $\mathrm{g}$ ai ha $\left.{ }^{-1}\right)$, trifluralin + halosulfuron $(600+35 \mathrm{~g}$ ai ha $\left.{ }^{-1}\right)$, ethalfluralin + halosulfuron $\left(810+35 \mathrm{~g}\right.$ ai ha $\left.^{-1}\right)$, pendimethalin + halo- 
sulfuron $\left(1080+35 \mathrm{~g}\right.$ ai ha $\left.{ }^{-1}\right)$, S-metolachlor + halosulfuron $(1050+35 \mathrm{~g}$ ai $\left.\mathrm{ha}^{-1}\right)$, dimethenamid-P + halosulfuron $\left(544+35 \mathrm{~g}^{\mathrm{ai} \mathrm{ha}} \mathrm{ha}^{-1}\right)$ and EPTC + halosulfuron $\left(3400+35 \mathrm{~g}\right.$ ai ha $\left.{ }^{-1}\right)$. Each plot was $3.0 \mathrm{~m}$ wide and consisted of 4 rows of “T9905" white beans spaced $0.75 \mathrm{~m}$ apart in rows that were $10 \mathrm{~m}$ long at Exeter and $8 \mathrm{~m}$ long at Ridgetown. White bean was planted at a rate of approximately 233,000 seeds ha ${ }^{-1}$ in late May to early June of each year.

Herbicide treatments were applied using a $\mathrm{CO}_{2}$-pressurized backpack sprayer calibrated to deliver $200 \mathrm{~L} \cdot \mathrm{ha}^{-1}$ aqueous solution at $240 \mathrm{kPa}$. The boom was 1.5 m long with four ultra-low drift (ULD 120-02, Pentair-Hypro, New Brighton, Minnesota) nozzles spaced $0.5 \mathrm{~m}$ apart, producing a spray width of $2.0 \mathrm{~m}$. Herbicide treatments were applied 1 - 2 days before planting and were immediately incorporated into the soil with two passes (in opposite directions) of an S-tine cultivator with rolling basket harrows. Weed-free plots were maintained weed-free during the growing season with trifluralin + halosulfuron $(600+25 \mathrm{~g}$ ai ha ${ }^{-1}$ ) applied PPI, hand hoeing, and hand weeding as required.

Crop injury was rated visually 2 and 4 weeks after white bean emergence (WAE) and weed control was assessed 4 and 8 WAE on a scale of $0 \%$ to $100 \%$ where a rating of 0 was defined as no injury/weed control and 100 was total crop/weed death. Weed density and shoot dry weight (biomass) were measured 8 WAE from two $0.25 \mathrm{~m}^{2}$ quadrats placed between the center two rows from each plot. White bean seed yield was measured at crop maturity by harvesting the middle two rows of each plot with a small-plot combine. Seed yield was adjusted to $18 \%$ seed moisture content for analysis.

Data analysis was completed using PROC GLIMMIX (SAS Ver. 9.4, SAS Institute Inc., Cary, NC). The model specified the fixed effect as herbicide treatment and random effects as year-location combinations (environment), environment by treatment interaction and replicate within environment. Distributions in PROC GLIMMIX were evaluated using fit statistics such as AICC and Chi-square/df ratio to check for overdispersion. Normality was assessed using the Shapiro-Wilk statistic and normal probability plot generated in PROC UNIVARIATE, and studentized residual plots generated in PROC GLIMMIX were used to check for obvious deviations from the assumption of variance homogeneity. In all cases, percent visible weed control evaluations best met the assumptions for analysis when arcsine-square root transformed prior to using a Gaussian distribution and identity link. Weed densities and dry biomass were analyzed using a log-normal distribution and identity link. Analysis was performed on the model scale, but for presentation purposes treatment means were back-transformed to the data scale, with a correction for log bias for density and dry biomass means. Tukey's HSD was employed to identify treatment differences at a significance level of 0.05 . In cases where a treatment had zero variance across all environments, it was excluded from the analysis; when the value of the treatment was zero, it could still be evaluated for differences with other treatments using the p-value generated in the LSMEANS output. 


\section{Results and Discussion}

\subsection{Weed Control}

Weeds analyzed needed to be present in two or more sites and included velvetleaf $(2 / 4)$, pigweed species $(2 / 4)$, common ragweed (3/4), common lambsquarters (4/4), wild mustard (2/4), barnyardgrass (4/4) and green foxtail (4/4). For pigweed species, Ridgetown had mostly green pigweed and Exeter had mostly redroot pigweed. Data were combined for analyses.

\subsubsection{Velvetleaf}

Trifluralin, ethalfluralin, pendimethalin, S-metolachlor and dimethenamid-P, applied PPI at rates evaluated, provided $0 \%$ control of velvetleaf with velvetleaf density and biomass similar to the weedy control (Table 1). EPTC and halosulfuron, applied PPI at rates evaluated, controlled velvetleaf $81-82$ and $98 \%$, respectively; velvetleaf density and biomass were similar to the weed-free control (Table 1). EPTC provided better velvetleaf control than the other grass herbicides evaluated. Trifluralin + halosulfuron, ethalfluralin + halosulfuron, pendimethalin + halosulfuron, S-metolachlor + halosulfuron, dimethenamid-P +

Table 1. Visible percent control 4 and 8 weeks after crop emergence (WAE), density and dry biomass 8 WAE for velvetleaf (ABUTH) with herbicides applied preplant incorporated in 2018 and 2019 at Ridgetown, Ontario.

\begin{tabular}{cccccc}
\hline \multirow{2}{*}{ Herbicide treatment } & Rate & \multicolumn{2}{c}{ ABUTH control } & ABUTH & ABUTH \\
\cline { 3 - 4 } & & 4 WAE & 8 WAE & density & dry biomass \\
\cline { 2 - 4 }$\left(\mathrm{g} \mathrm{ai} \mathrm{ha}^{-1}\right)$ & $(\%)$ & $(\%)$ & $\left(\right.$ plants $\left.^{-2}\right)$ & $\left(\mathrm{g} \mathrm{m}^{-2}\right)$ \\
\hline Weed-free control & & 100 & 100 & $0.0 \mathrm{a}$ & $0.0 \mathrm{a}$ \\
Weedy control & & $0 \mathrm{c}$ & $0 \mathrm{c}$ & $2.8 \mathrm{~cd}$ & $4.6 \mathrm{bc}$ \\
Trifluralin & 600 & $0 \mathrm{c}$ & $0 \mathrm{c}$ & $3.2 \mathrm{~d}$ & $19.9 \mathrm{c}$ \\
Ethalfluralin & 810 & $0 \mathrm{c}$ & $0 \mathrm{c}$ & $3.7 \mathrm{~d}$ & $29.1 \mathrm{c}$ \\
Pendimethalin & 1080 & $0 \mathrm{c}$ & $0 \mathrm{c}$ & $1.7 \mathrm{bcd}$ & $14.4 \mathrm{bc}$ \\
S-metolachlor & 1050 & $0 \mathrm{c}$ & $0 \mathrm{c}$ & $2.0 \mathrm{bcd}$ & $13.7 \mathrm{bc}$ \\
Dimethenamid-P & 544 & $0 \mathrm{c}$ & $0 \mathrm{c}$ & $1.8 \mathrm{bcd}$ & $11.1 \mathrm{bc}$ \\
EPTC & 3400 & $81 \mathrm{~b}$ & $82 \mathrm{~b}$ & $0.0 \mathrm{a}$ & $0.0 \mathrm{a}$ \\
Halosulfuron & 35 & $98 \mathrm{ab}$ & $98 \mathrm{ab}$ & $0.5 \mathrm{abc}$ & $0.6 \mathrm{ab}$ \\
Trifluralin + halosulfuron & $600+35$ & $97 \mathrm{ab}$ & $96 \mathrm{ab}$ & $0.2 \mathrm{ab}$ & $0.2 \mathrm{ab}$ \\
Ethalfluralin + halosulfuron & $810+35$ & $94 \mathrm{ab}$ & $93 \mathrm{ab}$ & $0.6 \mathrm{abc}$ & $1.2 \mathrm{~b}$ \\
Pendimethalin + halosulfuron & $1080+35$ & $98 \mathrm{ab}$ & $97 \mathrm{ab}$ & $0.5 \mathrm{abc}$ & $0.5 \mathrm{ab}$ \\
S-metolachlor + halosulfuron & $1050+35$ & $96 \mathrm{ab}$ & $97 \mathrm{ab}$ & $0.2 \mathrm{ab}$ & $0.2 \mathrm{ab}$ \\
Dimethenamid-P + halosulfuron & $544+35$ & $98 \mathrm{ab}$ & $98 \mathrm{ab}$ & $0.1 \mathrm{ab}$ & $0.2 \mathrm{ab}$ \\
EPTC + halosulfuron & $3400+35$ & $100 \mathrm{a}$ & $100 \mathrm{a}$ & $0.0 \mathrm{a}$ & $0.0 \mathrm{a}$ \\
\hline
\end{tabular}

Note: Means within a column followed by the same lowercase letter do not differ significantly according to Tukey's HSD at $\mathrm{p}<0.05$. 
halosulfuron, and EPTC + halosulfuron, applied PPI at rates evaluated controlled velvetleaf $93 \%$ - 100\%; velvetleaf density and biomass were similar to the weed-free control (Table 1). Results are similar to other studies in which most grass herbicides evaluated did not control velvetleaf but halosulfuron controlled velvetleaf up to $98 \%$ [11].

\subsubsection{Pigweed Species}

Trifluralin, ethalfluralin, pendimethalin, S-metolachlor, dimethenamid-P, EPTC, halosulfuron, trifluralin + halosulfuron, ethalfluralin + halosulfuron, pendimethalin + halosulfuron, S-metolachlor + halosulfuron, dimethenamid-P + halosulfuron, and EPTC + halosulfuron, applied PPI at rates evaluated, controlled pigweeds $87 \%-100 \%$ and reduced density $95 \%-100 \%$ and biomass $69 \%-100 \%$, compared to the weedy control (Table 2). These results are similar to other studies in which pigweed species were controlled $83 \%-100 \%$ with halosulfuron [9] [12] [13], 72\% - 98\% with trifluralin [9] [12] [14] [15], 91\% - 98\% with pendimethalin [9] [14] [15] [16], 84\% - 95\% with S-metolachlor [9] [12], 93\% - 97\% with dimethenamid-P [9] and 73\% - 78\% with EPTC applied PPI, in white beans [9] [15].

Table 2. Visible percent control 4 and 8 weeks after crop emergence (WAE), density and dry biomass 8 WAE for pigweed species (AMASS) with herbicides applied preplant incorporated in 2019 at Exeter and Ridgetown, Ontario.

\begin{tabular}{|c|c|c|c|c|c|}
\hline \multirow{3}{*}{ Herbicide treatment } & \multirow{2}{*}{ Rate } & \multicolumn{2}{|c|}{ AMASS control } & \multirow{2}{*}{$\begin{array}{l}\text { AMASS } \\
\text { density }\end{array}$} & \multirow{2}{*}{$\begin{array}{c}\text { AMASS } \\
\text { dry biomass }\end{array}$} \\
\hline & & $4 \mathrm{WAE}$ & $8 \mathrm{WAE}$ & & \\
\hline & $\left(\mathrm{g}\right.$ ai ha $\left.{ }^{-1}\right)$ & $(\%)$ & $(\%)$ & (plants $\mathrm{m}^{-2}$ ) & $\left(\mathrm{g} \mathrm{m}^{-2}\right)$ \\
\hline Weed-free control & & 100 & 100 & $0.0 \mathrm{a}$ & $0 \mathrm{a}$ \\
\hline Weedy control & & $0 \mathrm{c}$ & $0 \mathrm{c}$ & $46.0 \mathrm{e}$ & $223.7 \mathrm{e}$ \\
\hline Trifluralin & 600 & $97 \mathrm{ab}$ & $94 \mathrm{ab}$ & $2.0 \mathrm{~cd}$ & $26.4 \mathrm{bcd}$ \\
\hline Ethalfluralin & 810 & $99 \mathrm{ab}$ & $99 \mathrm{a}$ & $0.1 \mathrm{ab}$ & $1.0 \mathrm{abc}$ \\
\hline Pendimethalin & 1080 & $96 \mathrm{ab}$ & $96 \mathrm{ab}$ & $1.5 \mathrm{~cd}$ & $43.8 \mathrm{~cd}$ \\
\hline S-metolachlor & 1050 & $90 \mathrm{~b}$ & $87 \mathrm{~b}$ & $3.1 \mathrm{~d}$ & $70.4 \mathrm{~d}$ \\
\hline Dimethenamid-P & 544 & $98 \mathrm{ab}$ & $98 \mathrm{ab}$ & $1.1 \mathrm{bcd}$ & $21.9 \mathrm{bcd}$ \\
\hline EPTC & 3400 & $93 \mathrm{ab}$ & $95 \mathrm{ab}$ & $0.4 \mathrm{abc}$ & $2.8 \mathrm{abcd}$ \\
\hline Halosulfuron & 35 & $94 \mathrm{ab}$ & $94 \mathrm{ab}$ & $2.1 \mathrm{bcd}$ & $22.5 \mathrm{bcd}$ \\
\hline Trifluralin + halosulfuron & $600+35$ & $100 \mathrm{a}$ & 99 a & $0.0 \mathrm{a}$ & $0.0 \mathrm{a}$ \\
\hline Ethalfluralin + halosulfuron & $810+35$ & $100 \mathrm{a}$ & 99 a & $0.4 \mathrm{abc}$ & $3.5 \mathrm{abcd}$ \\
\hline Pendimethalin + halosulfuron & $1080+35$ & $100 \mathrm{a}$ & $100 \mathrm{a}$ & $0.1 \mathrm{ab}$ & $0.1 \mathrm{ab}$ \\
\hline S-metolachlor + halosulfuron & $1050+35$ & $99 \mathrm{ab}$ & $99 \mathrm{a}$ & $0.0 \mathrm{a}$ & $0.0 \mathrm{a}$ \\
\hline Dimethenamid-P + halosulfuron & $544+35$ & $99 \mathrm{ab}$ & 99 a & $0.0 \mathrm{a}$ & $0.0 \mathrm{a}$ \\
\hline EPTC + halosulfuron & $3400+35$ & $100 \mathrm{a}$ & $100 \mathrm{a}$ & $0.0 \mathrm{a}$ & $0.0 \mathrm{a}$ \\
\hline
\end{tabular}

Note: Means within a column followed by the same lowercase letter do not differ significantly according to Tukey's HSD at $\mathrm{p}<0.05$. 


\subsubsection{Common Ragweed}

Trifluralin, ethalfluralin, pendimethalin, S-metolachlor and dimethenamid-P, applied PPI at rates evaluated, controlled common ragweed only $0 \%-41 \%$; common ragweed density and biomass were similar to the weedy control (Table 3). EPTC and halosulfuron, applied PPI at rates evaluated, provided $85 \%-93 \%$ and $90 \%-94 \%$ control of common ragweed and reduced density $78 \%$ and $94 \%$ and biomass 85 and 93\%, respectively (Table 3). EPTC provided better control of common ragweed than the other grass herbicides evaluated. Trifluralin + halosulfuron, ethalfluralin + halosulfuron, pendimethalin + halosulfuron, S-metolachlor + halosulfuron, dimethenamid-P + halosulfuron and EPTC + halosulfuron, applied PPI at rates evaluated, controlled common ragweed $88 \%-99 \%$ and reduced common ragweed density $92 \%-97 \%$ and biomass $60 \%-98 \%$, compared to the weedy control (Table 3). In other studies, common ragweed was controlled 95\% - 99\% with halosulfuron [9] [12], 9\% - 28\% with trifluralin [9] [12] [14] [15] [17], 1\% - 13\% with pendimethalin [9] [14] [15] [16], 13\% - 40\% with S-metolachlor [9] [12], 41\% - 56\% with dimethenamid-P [8] and 52\% - 71\% with EPTC [9] [15], applied PPI, in white beans.

Table 3. Visible percent control 4 and 8 weeks after crop emergence (WAE), density and dry biomass 8 WAE for common ragweed (AMBEL) with herbicides applied preplant incorporated in 2019 at Exeter and 2018 and 2019 at Ridgetown, Ontario.

\begin{tabular}{|c|c|c|c|c|c|}
\hline \multirow{3}{*}{ Herbicide treatment } & \multirow{2}{*}{ Rate } & \multicolumn{2}{|c|}{ AMBEL control } & \multirow{2}{*}{$\begin{array}{c}\text { AMBEL } \\
\text { density }\end{array}$} & \multirow{2}{*}{$\begin{array}{c}\text { AMBEL } \\
\text { dry biomass }\end{array}$} \\
\hline & & $4 \mathrm{WAE}$ & 8 WAE & & \\
\hline & $\left(\mathrm{g} \mathrm{ai} \mathrm{ha}^{-1}\right)$ & (\%) & (\%) & (plants $\mathrm{m}^{-2}$ ) & $\left(\mathrm{g} \mathrm{m}^{-2}\right)$ \\
\hline Weed-free control & & 100 & 100 & $0 \mathrm{a}$ & $0 \mathrm{a}$ \\
\hline Weedy control & & $0 \mathrm{~d}$ & $0 \mathrm{c}$ & $31.1 \mathrm{e}$ & $101.5 \mathrm{e}$ \\
\hline Trifluralin & 600 & $2 c$ & $0 \mathrm{c}$ & $27.2 \mathrm{e}$ & $212.3 \mathrm{e}$ \\
\hline Ethalfluralin & 810 & $2 \mathrm{~cd}$ & $1 \mathrm{c}$ & $31.0 \mathrm{e}$ & $150.7 \mathrm{e}$ \\
\hline Pendimethalin & 1080 & $9 c$ & $0 \mathrm{c}$ & $26.4 \mathrm{de}$ & $197.0 \mathrm{e}$ \\
\hline S-metolachlor & 1050 & $7 c$ & $3 \mathrm{bc}$ & $24.8 \mathrm{de}$ & $176.7 \mathrm{e}$ \\
\hline Dimethenamid-P & 544 & $41 \mathrm{~b}$ & $25 \mathrm{~b}$ & $19.0 \mathrm{cde}$ & 99.6 de \\
\hline EPTC & 3400 & $93 \mathrm{a}$ & $85 \mathrm{a}$ & $6.8 \mathrm{bcd}$ & $15.0 \mathrm{bcd}$ \\
\hline Halosulfuron & 35 & 94 a & $90 \mathrm{a}$ & $1.9 \mathrm{ab}$ & $6.6 \mathrm{bc}$ \\
\hline Trifluralin + halosulfuron & $600+35$ & $94 \mathrm{a}$ & $93 \mathrm{a}$ & $1.2 \mathrm{ab}$ & $9.4 \mathrm{bc}$ \\
\hline Ethalfluralin + halosulfuron & $810+35$ & $92 \mathrm{a}$ & $90 \mathrm{a}$ & $2.5 \mathrm{bc}$ & $16.9 \mathrm{bcd}$ \\
\hline Pendimethalin + halosulfuron & $1080+35$ & $92 \mathrm{a}$ & 88 a & $1.9 \mathrm{ab}$ & $40.7 \mathrm{~cd}$ \\
\hline S-metolachlor + halosulfuron & $1050+35$ & 96 a & $91 \mathrm{a}$ & $2.2 \mathrm{bc}$ & $49.1 \mathrm{~cd}$ \\
\hline Dimethenamid-P + halosulfuron & $544+35$ & 97 a & 94 a & $1.0 \mathrm{ab}$ & $6.3 \mathrm{bc}$ \\
\hline EPTC + halosulfuron & $3400+35$ & $99 a$ & $98 \mathrm{a}$ & $0.9 \mathrm{ab}$ & $2.5 \mathrm{ab}$ \\
\hline
\end{tabular}

Note: Means within a column followed by the same lowercase letter do not differ significantly according to Tukey's HSD at $\mathrm{p}<0.05$. 


\subsubsection{Common Lambsquarters}

Trifluralin, ethalfluralin, pendimethalin, S-metolachlor, dimethenamid-P, EPTC and halosulfuron, applied PPI at rates evaluated, controlled common lambsquarters $81 \%-99 \%$ and reduced density $75 \%-100 \%$ and biomass $71 \%-$ $100 \%$ (Table 4). Trifluralin + halosulfuron, ethalfluralin + halosulfuron, pendimethalin + halosulfuron, S-metolachlor + halosulfuron, dimethenamid-P + halosulfuron, and EPTC + halosulfuron, applied PPI at rates evaluated, controlled common lambsquarters $99 \%-100 \%$ and reduced common lambsquarters density $98 \%-100 \%$ and biomass $97 \%-100 \%$, compared to the weedy control (Table 4). Results are similar to other studies in which common lambsquarters was controlled $96 \%-100 \%$ with halosulfuron [9] [12] [13], $60 \%$ - 92\% with trifluralin [9] [12] [13] [14] [15] [17], 56\% - 97\% with pendimethalin [9] [14] [15] [16], 19\% - 82\% with S-metolachlor [9] [12], 55\% - 72\% with dimethenamid-P [9] and 77\% - 85\% with EPTC [9] [15], applied PPI, in white beans.

\subsubsection{Wild Mustard}

Trifluralin, ethalfluralin, pendimethalin, S-metolachlor and dimethenamid-P, applied PPI at rates evaluated, controlled wild mustard 0\% - 46\%; wild mustard

Table 4. Visible percent control 4 and 8 weeks after crop emergence (WAE), density and dry biomass 8 WAE for common lambsquarters (CHEAL) with herbicides applied preplant incorporated in 2017 and 2019 at Exeter and 2018 and 2019 at Ridgetown, Ontario.

\begin{tabular}{|c|c|c|c|c|c|}
\hline \multirow{3}{*}{ Herbicide treatment } & \multirow{2}{*}{ Rate } & \multicolumn{2}{|c|}{ CHEAL control } & \multirow{2}{*}{$\begin{array}{l}\text { CHEAL } \\
\text { density }\end{array}$} & \multirow{2}{*}{$\begin{array}{c}\text { CHEAL } \\
\text { dry biomass }\end{array}$} \\
\hline & & $4 \mathrm{WAE}$ & $8 \mathrm{WAE}$ & & \\
\hline & $\left(\mathrm{g} \mathrm{ai} \mathrm{ha}^{-1}\right)$ & (\%) & $(\%)$ & (plants $\mathrm{m}^{-2}$ ) & $\left(\mathrm{g} \mathrm{m}^{-2}\right)$ \\
\hline Weed-free control & & 100 & 100 & $0 \mathrm{a}$ & $0 \mathrm{a}$ \\
\hline Weedy control & & $0 \mathrm{~d}$ & $0 \mathrm{~d}$ & $25.3 \mathrm{e}$ & $88.3 \mathrm{~b}$ \\
\hline Trifluralin & 600 & $97 \mathrm{ab}$ & $98 \mathrm{ab}$ & $0.7 \mathrm{abc}$ & $9.5 \mathrm{ab}$ \\
\hline Ethalfluralin & 810 & 99 a & $99 \mathrm{a}$ & $0.1 \mathrm{ab}$ & $0.1 \mathrm{a}$ \\
\hline Pendimethalin & 1080 & $97 \mathrm{ab}$ & 99 a & $1.1 \mathrm{bc}$ & $12.7 \mathrm{ab}$ \\
\hline S-metolachlor & 1050 & $81 \mathrm{c}$ & $83 \mathrm{c}$ & $6.2 \mathrm{~d}$ & $25.6 \mathrm{~b}$ \\
\hline Dimethenamid-P & 544 & $88 \mathrm{bc}$ & $88 \mathrm{bc}$ & $3.6 \mathrm{~cd}$ & $3.8 \mathrm{ab}$ \\
\hline EPTC & 3400 & $98 \mathrm{a}$ & $97 \mathrm{ab}$ & $0.8 \mathrm{abc}$ & $0.2 \mathrm{a}$ \\
\hline Halosulfuron & 35 & $97 \mathrm{ab}$ & $97 \mathrm{ab}$ & $1.8 \mathrm{bcd}$ & $15.7 \mathrm{ab}$ \\
\hline Trifluralin + halosulfuron & $600+35$ & 99 a & $100 \mathrm{a}$ & $0.4 \mathrm{abc}$ & $3.0 \mathrm{ab}$ \\
\hline Ethalfluralin + halosulfuron & $810+35$ & $100 \mathrm{a}$ & $100 \mathrm{a}$ & $0.1 \mathrm{ab}$ & $0.0 \mathrm{a}$ \\
\hline Pendimethalin + halosulfuron & $1080+35$ & $100 \mathrm{a}$ & $100 \mathrm{a}$ & $0.2 \mathrm{ab}$ & $0.0 \mathrm{a}$ \\
\hline S-metolachlor + halosulfuron & $1050+35$ & 99 a & $99 \mathrm{a}$ & $0.1 \mathrm{ab}$ & $0.1 \mathrm{a}$ \\
\hline Dimethenamid-P + halosulfuron & $544+35$ & $100 \mathrm{a}$ & $100 \mathrm{a}$ & $0.2 \mathrm{ab}$ & $0.0 \mathrm{a}$ \\
\hline EPTC + halosulfuron & $3400+35$ & $100 \mathrm{a}$ & $100 \mathrm{a}$ & $0.0 \mathrm{a}$ & $0.0 \mathrm{a}$ \\
\hline
\end{tabular}

Note: Means within a column followed by the same lowercase letter do not differ significantly according to Tukey's HSD at $\mathrm{p}<0.05$. 
density and biomass were similar to the weedy control (Table 5). EPTC provided better control of wild mustard than the other grass herbicides evaluated. EPTC applied PPI at the rate evaluated provided $71 \%$ control of wild mustard; wild mustard density and biomass were similar to the weed-free control (Table 5). Halosulfuron alone or in combination with trifluralin, ethalfluralin, pendimethalin, S-metolachlor, dimethenamid-P and EPTC, applied PPI at rates evaluated, controlled wild mustard $97 \%-100 \%$ and reduced density $98 \%-100 \%$ and biomass 99\% - 100\% (Table 5). In other studies, wild mustard was controlled 99\% - 100\% with halosulfuron [9] [12] [13], 11\% - 44\% with trifluralin [9] [12] [14] [15], $0 \%-23 \%$ with pendimethalin [9] [12] [15] [16], $11 \%-55 \%$ with S-metolachlor [9] [12], 27\% - 70\% with dimethenamid-P [9] and 24\% - 68\% with EPTC [9] [15], applied PPI in white beans.

\subsubsection{Barnyardgrass}

Trifluralin, ethalfluralin, pendimethalin, S-metolachlor, dimethenamid-P and EPTC, applied PPI at rates evaluated, controlled barnyardgrass $98 \%-100 \%$ and reduced density $95 \%$ - 99\% and biomass $98 \%$ - 100\% (Table 6). Halosulfuron controlled barnyardgrass up to $24 \%$; density and biomass were similar to the

Table 5. Visible percent control 4 and 8 weeks after crop emergence (WAE), density and dry biomass 8 WAE for wild mustard (SINAR) with herbicides applied preplant incorporated in 2017 and 2019 at Exeter, Ontario.

\begin{tabular}{|c|c|c|c|c|c|}
\hline \multirow{3}{*}{ Herbicide treatment } & \multirow{2}{*}{ Rate } & \multicolumn{2}{|c|}{ SINAR control } & \multirow{2}{*}{$\begin{array}{l}\text { SINAR } \\
\text { density }\end{array}$} & \multirow{2}{*}{$\begin{array}{c}\text { SINAR } \\
\text { dry biomass }\end{array}$} \\
\hline & & $4 \mathrm{WAE}$ & $8 \mathrm{WAE}$ & & \\
\hline & $\left(\mathrm{g}\right.$ ai ha $\left.{ }^{-1}\right)$ & $(\%)$ & $(\%)$ & (plants $\mathrm{m}^{-2}$ ) & $\left(\mathrm{g} \mathrm{m}^{-2}\right)$ \\
\hline Weed-free control & & 100 & 100 & $0 \mathrm{a}$ & $0 \mathrm{a}$ \\
\hline Weedy control & & $0 \mathrm{e}$ & $0 \mathrm{c}$ & $128.0 \mathrm{de}$ & $135.3 \mathrm{c}$ \\
\hline Trifluralin & 600 & $11 \mathrm{~d}$ & $3 c$ & $136.2 \mathrm{de}$ & $181.0 \mathrm{c}$ \\
\hline Ethalfluralin & 810 & $16 \mathrm{~cd}$ & $8 \mathrm{bc}$ & 115.6 bcde & $164.8 \mathrm{c}$ \\
\hline Pendimethalin & 1080 & $0 \mathrm{e}$ & $0 \mathrm{c}$ & $127.4 \mathrm{cde}$ & $224.2 \mathrm{c}$ \\
\hline S-metolachlor & 1050 & $20 \mathrm{~cd}$ & $3 c$ & $156.2 \mathrm{e}$ & $176.1 \mathrm{c}$ \\
\hline Dimethenamid-P & 544 & $46 \mathrm{bc}$ & $8 \mathrm{bc}$ & 85.1 bcde & $69.8 c$ \\
\hline EPTC & 3400 & $71 \mathrm{~b}$ & $71 \mathrm{ab}$ & 97.3 bcde & $54.5 \mathrm{bc}$ \\
\hline Halosulfuron & 35 & $98 \mathrm{a}$ & $100 \mathrm{a}$ & $0.1 \mathrm{ab}$ & $0.0 \mathrm{a}$ \\
\hline Trifluralin + halosulfuron & $600+35$ & $97 \mathrm{a}$ & $100 \mathrm{a}$ & $0.2 \mathrm{ab}$ & $0.0 \mathrm{a}$ \\
\hline Ethalfluralin + halosulfuron & $810+35$ & $98 \mathrm{a}$ & 99 a & $0.4 \mathrm{abc}$ & $0.5 \mathrm{ab}$ \\
\hline Pendimethalin + halosulfuron & $1080+35$ & 99 a & $100 \mathrm{a}$ & $0.0 \mathrm{a}$ & $0.0 \mathrm{a}$ \\
\hline S-metolachlor + halosulfuron & $1050+35$ & $99 \mathrm{a}$ & $100 \mathrm{a}$ & $0.0 \mathrm{a}$ & $0.0 \mathrm{a}$ \\
\hline Dimethenamid-P + halosulfuron & $544+35$ & $99 \mathrm{a}$ & $100 \mathrm{a}$ & $2.0 \mathrm{abcd}$ & $1.3 \mathrm{ab}$ \\
\hline EPTC + halosulfuron & $3400+35$ & $99 \mathrm{a}$ & $100 \mathrm{a}$ & $1.3 \mathrm{abcd}$ & $0.3 \mathrm{ab}$ \\
\hline
\end{tabular}

Note: Means within a column followed by the same lowercase letter do not differ significantly according to Tukey's HSD at $\mathrm{p}<0.05$. 
Table 6. Visible percent control 4 and 8 weeks after crop emergence (WAE), density and dry biomass 8 WAE for barnyardgrass (ECHCG) with herbicides applied preplant incorporated in 2017 and 2019 at Exeter and 2018 and 2019 at Ridgetown, Ontario.

\begin{tabular}{|c|c|c|c|c|c|}
\hline \multirow{3}{*}{ Herbicide treatment } & \multirow{2}{*}{ Rate } & \multicolumn{2}{|c|}{ ECHCG control } & \multirow{2}{*}{$\begin{array}{c}\text { ECHCG } \\
\text { density }\end{array}$} & \multirow{2}{*}{$\begin{array}{c}\text { ECHCG } \\
\text { dry biomass }\end{array}$} \\
\hline & & $4 \mathrm{WAE}$ & $8 \mathrm{WAE}$ & & \\
\hline & $\left(\mathrm{g}_{\mathrm{ai} \mathrm{ha}}{ }^{-1}\right)$ & $(\%)$ & (\%) & (plants $\mathrm{m}^{-2}$ ) & $\left(\mathrm{g} \mathrm{m}^{-2}\right)$ \\
\hline Weed-free control & & 100 & 100 & $0 \mathrm{a}$ & $0 \mathrm{a}$ \\
\hline Weedy control & & $0 \mathrm{c}$ & $0 \mathrm{c}$ & $22.0 \mathrm{c}$ & $152.8 \mathrm{c}$ \\
\hline Trifluralin & 600 & $99 \mathrm{a}$ & $99 \mathrm{a}$ & $1.0 \mathrm{~b}$ & $2.7 \mathrm{ab}$ \\
\hline Ethalfluralin & 810 & $99 \mathrm{a}$ & $99 \mathrm{a}$ & $0.3 \mathrm{ab}$ & $1.2 \mathrm{ab}$ \\
\hline Pendimethalin & 1080 & $99 \mathrm{a}$ & $99 \mathrm{a}$ & $0.7 \mathrm{ab}$ & $2.3 \mathrm{ab}$ \\
\hline S-metolachlor & 1050 & $99 \mathrm{a}$ & $99 \mathrm{a}$ & $0.9 \mathrm{ab}$ & $1.8 \mathrm{ab}$ \\
\hline Dimethenamid-P & 544 & $98 \mathrm{a}$ & $99 \mathrm{a}$ & $0.9 \mathrm{ab}$ & $1.6 \mathrm{ab}$ \\
\hline EPTC & 3400 & $100 \mathrm{a}$ & $100 \mathrm{a}$ & $0.3 \mathrm{ab}$ & $0.7 \mathrm{ab}$ \\
\hline Halosulfuron & 35 & $19 \mathrm{~b}$ & $24 \mathrm{~b}$ & $19.0 \mathrm{c}$ & $135.4 \mathrm{c}$ \\
\hline Trifluralin + halosulfuron & $600+35$ & $99 \mathrm{a}$ & $98 \mathrm{a}$ & $0.5 \mathrm{ab}$ & $6.3 \mathrm{ab}$ \\
\hline Ethalfluralin + halosulfuron & $810+35$ & $99 \mathrm{a}$ & 99 a & $1.6 \mathrm{~b}$ & $10.9 \mathrm{~b}$ \\
\hline Pendimethalin + halosulfuron & $1080+35$ & $98 \mathrm{a}$ & $99 \mathrm{a}$ & $1.2 \mathrm{~b}$ & $3.2 \mathrm{ab}$ \\
\hline S-metolachlor + halosulfuron & $1050+35$ & $97 \mathrm{a}$ & $98 \mathrm{a}$ & $0.9 \mathrm{ab}$ & $6.7 \mathrm{ab}$ \\
\hline Dimethenamid-P + halosulfuron & $544+35$ & $99 \mathrm{a}$ & $99 \mathrm{a}$ & $1.5 \mathrm{~b}$ & $4.1 \mathrm{ab}$ \\
\hline EPTC + halosulfuron & $3400+35$ & $99 \mathrm{a}$ & $99 \mathrm{a}$ & $1.6 \mathrm{~b}$ & $1.0 \mathrm{ab}$ \\
\hline
\end{tabular}

Note: Means within a column followed by the same lowercase letter do not differ significantly according to Tukey's HSD at $\mathrm{p}<0.05$.

weedy control (Table 6). However, halosulfuron in combination with trifluralin, ethalfluralin, pendimethalin, S-metolachlor, dimethenamid-P or EPTC, applied PPI at rates evaluated, provided $97 \%$ - $99 \%$ control of barnyardgrass and reduced density $93 \%$ - 98\% and biomass $93 \%$ - 99\% (Table 6). In other studies, soil application of trifluralin provided excellent control ( $>90 \%)$ of barnyardgrass, but pendimethalin provided only $58 \%$ control of barnyardgrass in white beans [9] [14].

\subsubsection{Green Foxtail}

Trifluralin, ethalfluralin, pendimethalin, S-metolachlor, dimethenamid-P and EPTC, applied PPI at rates evaluated, controlled green foxtail $98 \%-99 \%$ and reduced density $95 \%$ - 99\% and biomass $88 \%$ - 99\% (Table 7). Halosulfuron, applied PPI at the rate evaluated, provided only up to $25 \%$ control of green foxtail; density and biomass were similar to the weedy control (Table 7). Trifluralin + halosulfuron, ethalfluralin + halosulfuron, pendimethalin + halosulfuron, S-metolachlor + halosulfuron, dimethenamid-P + halosulfuron, and EPTC + halosulfuron, applied PPI at rates evaluated, provided 94\% - 99\% control of green foxtail and reduced green foxtail density $89 \%$ - 97\% and biomass 53\%- 
Table 7. Visible percent control 4 and 8 weeks after crop emergence (WAE), density and dry biomass 8 WAE for green foxtail (SETVI) with herbicides applied preplant incorporated in 2017 and 2019 at Exeter and 2018 and 2019 at Ridgetown, Ontario.

\begin{tabular}{|c|c|c|c|c|c|}
\hline \multirow{3}{*}{ Herbicide treatment } & \multirow{2}{*}{ Rate } & \multicolumn{2}{|c|}{ SETVI control } & \multirow{2}{*}{$\begin{array}{l}\text { SETVI } \\
\text { density }\end{array}$} & \multirow{2}{*}{$\begin{array}{c}\text { SETVI } \\
\text { dry biomass }\end{array}$} \\
\hline & & $4 \mathrm{WAE}$ & $8 \mathrm{WAE}$ & & \\
\hline & $\left(\mathrm{g}\right.$ ai ha $\left.{ }^{-1}\right)$ & (\%) & (\%) & $\left(\right.$ plants $\mathrm{m}^{-2}$ ) & $\left(\mathrm{g} \mathrm{m}^{-2}\right)$ \\
\hline Weed-free control & & 100 & 100 & $0 \mathrm{a}$ & $0 \mathrm{a}$ \\
\hline Weedy control & & $0 \mathrm{c}$ & $0 \mathrm{c}$ & $129.2 \mathrm{e}$ & $184.6 \mathrm{e}$ \\
\hline Trifluralin & 600 & $98 \mathrm{a}$ & $98 \mathrm{a}$ & $5.8 \mathrm{bcd}$ & $22.1 \mathrm{bcd}$ \\
\hline Ethalfluralin & 810 & $99 \mathrm{a}$ & $99 \mathrm{a}$ & $0.7 \mathrm{ab}$ & $1.0 \mathrm{ab}$ \\
\hline Pendimethalin & 1080 & $98 \mathrm{a}$ & $98 \mathrm{a}$ & $4.1 \mathrm{bc}$ & $19.9 \mathrm{bcd}$ \\
\hline S-metolachlor & 1050 & $98 \mathrm{a}$ & $98 \mathrm{a}$ & $6.7 \mathrm{~cd}$ & $10.2 \mathrm{bcd}$ \\
\hline Dimethenamid-P & 544 & $99 \mathrm{a}$ & $99 \mathrm{a}$ & $5.4 \mathrm{bcd}$ & $7.6 \mathrm{bc}$ \\
\hline EPTC & 3400 & $99 \mathrm{a}$ & $98 \mathrm{a}$ & $6.4 \mathrm{bcd}$ & $3.2 \mathrm{bc}$ \\
\hline Halosulfuron & 35 & $25 \mathrm{~b}$ & $20 \mathrm{~b}$ & $65.5 \mathrm{e}$ & $140.7 \mathrm{e}$ \\
\hline Trifluralin + halosulfuron & $600+35$ & $95 \mathrm{a}$ & $95 \mathrm{a}$ & $11.2 \mathrm{~cd}$ & $79.2 \mathrm{~cd}$ \\
\hline Ethalfluralin + halosulfuron & $810+35$ & $98 \mathrm{a}$ & $99 \mathrm{a}$ & $4.2 \mathrm{bc}$ & $3.0 \mathrm{abc}$ \\
\hline Pendimethalin + halosulfuron & $1080+35$ & $96 \mathrm{a}$ & $97 \mathrm{a}$ & $7.3 \mathrm{bcd}$ & $11.5 \mathrm{bcd}$ \\
\hline S-metolachlor + halosulfuron & $1050+35$ & $95 \mathrm{a}$ & $94 \mathrm{a}$ & $14.2 \mathrm{~d}$ & $87.4 \mathrm{~d}$ \\
\hline Dimethenamid-P + halosulfuron & $544+35$ & $97 \mathrm{a}$ & $97 \mathrm{a}$ & $6.0 \mathrm{bcd}$ & $6.9 \mathrm{bcd}$ \\
\hline EPTC + halosulfuron & $3400+35$ & $98 \mathrm{a}$ & $97 \mathrm{a}$ & $8.2 \mathrm{bcd}$ & $11.5 \mathrm{bcd}$ \\
\hline
\end{tabular}

Note: Means within a column followed by the same lowercase letter do not differ significantly according to Tukey's HSD at $\mathrm{p}<0.05$.

98\% (Table 7). Results are similar to other studies in which halosulfuron, trifluralin, pendimethalin, S-metolachlor, dimethenamid-P and EPTC, applied PPI, provided 47\% - 59\% [9] [12], 94\% - 100\% [9] [12] [14] [15] [17], 92\% - 98\% [9] [16], 93\% - 97\% [9] [12], 95\% - 96\% [9] and 94\% - 99\% [9] [15] control of green foxtail in white beans, respectively.

\subsection{Crop Injury and Seed Yield}

White bean injury for all treatments evaluated was zero and was not analyzed (data not shown). Weed interference reduced white bean seed yield by $70 \%$ (Table 8). White bean seed yield was $53 \%-66 \%$ of the weed-free control with trifluralin, ethalfluralin, pendimethalin, S-metolachlor and dimethenamid-P (Table 8). White bean seed yield was $81 \%$ and $58 \%$ of the weed-free control with EPTC and halosulfuron, applied PPI, respectively (Table 8). Trifluralin + halosulfuron, ethalfluralin + halosulfuron, pendimethalin + halosulfuron, S-metolachlor + halosulfuron, dimethenamid-P + halosulfuron, and EPTC + halosulfuron, applied PPI at rates evaluated, resulted in white bean seed yield that was $87 \%-95 \%$ of the weed-free control (Table 8). There was no herbicide treatment effect on the seed moisture content which indicates no delay in white 
Table 8. White bean moisture at harvest and yield with herbicide treatments applied preplant incorporated in 2017 and 2019 at Exeter and 2018 and 2019 at Ridgetown, Ontario.

\begin{tabular}{cccc}
\hline \multirow{2}{*}{ Herbicide treatment } & Rate & White bean moisture & White bean yield \\
\cline { 2 - 4 }$\left(\mathrm{g} \mathrm{ai} \mathrm{ha}^{-1}\right)$ & (\% of weed-free control) \\
\hline Weedy control & 600 & $104 \mathrm{a}$ & $30 \mathrm{e}$ \\
Trifluralin & 810 & $103 \mathrm{a}$ & $53 \mathrm{de}$ \\
Ethalfluralin & 1080 & $103 \mathrm{a}$ & $56 \mathrm{~cd}$ \\
Pendimethalin & 1050 & $104 \mathrm{a}$ & $53 \mathrm{de}$ \\
S-metolachlor & 544 & $102 \mathrm{a}$ & $58 \mathrm{~cd}$ \\
Dimethenamid-P & 3400 & $103 \mathrm{a}$ & $66 \mathrm{bcd}$ \\
EPTC & 35 & $99 \mathrm{a}$ & $81 \mathrm{abc}$ \\
Halosulfuron & $600+35$ & $102 \mathrm{a}$ & $58 \mathrm{~cd}$ \\
Trifluralin + halosulfuron & $810+35$ & $100 \mathrm{a}$ & $88 \mathrm{ab}$ \\
Ethalfluralin + halosulfuron & $1080+35$ & $102 \mathrm{a}$ & $89 \mathrm{ab}$ \\
Pendimethalin + halosulfuron & $1050+35$ & $101 \mathrm{a}$ & $91 \mathrm{ab}$ \\
S-metolachlor + halosulfuron & $544+35$ & $103 \mathrm{a}$ & $87 \mathrm{ab}$ \\
Dimethenamid-P + halosulfuron & $3400+35$ & $102 \mathrm{a}$ & $94 \mathrm{a}$ \\
EPTC + halosulfuron & & $95 \mathrm{a}$ \\
\hline
\end{tabular}

Note: Means within a column followed by the same lowercase letter do not differ significantly according to Tukey's HSD at $\mathrm{p}<0.05$.

bean maturity with any of the herbicide treatments evaluated (Table 8). Results are similar to other studies in which weed interference with halosulfuron, pendimethalin, dimethenamid-P and S-metolachlor herbicide treatments reduced white bean seed yield $20 \%, 66 \%, 72 \%$ and $62 \%$, respectively [9] [10].

\section{Conclusion}

Results indicate that halosulfuron provides excellent control of velvetleaf, pigweed species, common ragweed, common lambsquarters, and wild mustard and minimal control of barnyardgrass and green foxtail. Trifluralin, ethalfluralin, pendimethalin, S-metolachlor and dimethenamid-P provide minimal control of velvetleaf, common ragweed, wild mustard and good to excellent control of pigweed species, common lambsquarters, barnyardgrass and green foxtail. EPTC provides fair to good control of velvetleaf and wild mustard and good to excellent control of pigweeds, common ragweed, common lambsquarters, barnyardgrass and green foxtail. EPTC provides better control of velvetleaf, common ragweed and wild mustard than the other grass herbicides evaluated. Halosulfuron tankmixed with trifluralin, ethalfluralin, pendimethalin, S-metolachlor, dimethenamid-P and EPTC provides excellent control of velvetleaf, pigweeds, common ragweed, common lambsquarters, wild mustard, barnyardgrass and green foxtail. Ethalfluralin provides comparable weed control as the other grass 
herbicides evaluated, and has potential to be registered for the control of grass weed species in white beans. Based on these results, halosulfuron in combination with any of the grass herbicides evaluated can provide effective broad-spectrum control of common annual grass and broadleaf weeds in dry bean production in Ontario, Canada.

\section{Acknowledgements}

Funding for this project was provided in part by the Ontario Bean Growers (OBG).

\section{Conflicts of Interest}

The authors declare no conflicts of interest regarding the publication of this paper.

\section{References}

[1] [OMAFRA] Ontario Ministry of Agriculture and Food and Rural Affairs (2017) Area, Yield, Production and Farm Value of Specified Field Crops, Ontario, 2012-2017.

[2] Soltani, N., Dille, J.A., Burke, I.C., Everman, W.J., VanGessel, M.J., Davis, V.M. and Sikkema, P.H. (2018) Potential Yield Loss in Dry Bean Crops Due to Weeds in the United States and Canada. Weed Technology, 32, 342-346. https://doi.org/10.1017/wet.2017.116

[3] Soltani, N., Dille, J.A., Burke, I.C., Everman, W.J., VanGessel, M.J., Davis, V.M. and Sikkema P.H. (2017) Perspectives on Potential Soybean Yield Losses from Weeds in North America. Weed Technology, 31, 148-154. https://doi.org/10.1017/wet.2016.2

[4] Soltani, N., Dille, J.A., Burke, I.C., Everman, W.J., VanGessel, M.J., Davis, V.M. and Sikkema, P.H. (2016) Potential Corn Yield Losses from Weeds in North America. Weed Technology, 30, 979-984. https://doi.org/10.1614/WT-D-16-00046.1

[5] [OMAFRA] Ontario Ministry of Agriculture and Food and Rural Affairs (2020) Guide to Weed Control, Publication 75. Toronto, 1-457.

[6] Duggleby, R.G., McCourt, J.A. and Guddat, L.W. (2008) Structure and Mechanism of Inhibition of Plant Acetohydroxyacid Synthase. Plant Physiology and Biochemistry, 46, 309-324. https://doi.org/10.1016/j.plaphy.2007.12.004

[7] Buker, R.S., Stall, W.M. and Olson, S.M. (1998) Watermelon Tolerance to Halosulfuron Applied Preemergence and Postemergence. Proceedings of Annual Meeting of the Florida State Horticulture Society, Vol. 110, 323-325.

[8] Li, Z., Van Acker, R.C., Robinson, D.E., Soltani, N. and Sikkema, P.H. (2016) Halosulfuron Tank-Mixes Applied PRE in White Bean. Weed Technology, 30, 57-66. https://doi.org/10.1614/WT-D-15-00084.1

[9] Li, Z., Van Acker, R.C., Robinson, D.E., Soltani, N. and Sikkema, P.H. (2016) Halosulfuron Tankmixes Applied Preplant Incorporated for Weed Control in White Bean (Phaseolus vulgaris L.). Canadian Journal of Plant Science, 96, 481-488. https://doi.org/10.1139/cjps-2015-0124

[10] Shaner, D. (2014) Herbicide Handbook. 10th Edition, Weed Science, Champaign.

[11] Brown, D. and Masiunas, J. (2002) Evaluation of Herbicides for Pumpkin (Cucurbita spp.). Weed Technology, 16, 282-292. 
https://doi.org/10.1614/0890-037X(2002)016[0282:EOHFPC]2.0.CO;2

[12] Soltani, N., Nurse, R.E., Shropshire, C. and Sikkema, P.H. (2014) Weed Control in White Bean with Various Halosulfuron Tankmixes. Advances in Agriculture, 2014, Article ID: 391634. https://doi.org/10.1155/2014/391634

[13] Soltani, N., Nurse, R.E., Shropshire, C. and Sikkema, P.H. (2014) Weed Control with Halosulfuron Applied Preplant Incorporated, Preemergence or Postemergence in White Bean. Agricultural Science, 5, 875-881. https://doi.org/10.4236/as.2014.510094

[14] Soltani, N., Nurse, R.E. and Sikkema, P.H. (2012) Weed Control in Dry Bean with Pendimethalin plus Reduced Rates of Imazethapyr. International Research Journal of Agricultural Science and Soil Science, 2, 312-317.

[15] Soltani, N., Nurse, R.E., Shropshire, C. and Sikkema, P.H. (2012) Weed Control, Environmental Impact and Profitability of Pre-Plant Incorporated Herbicides in White Bean. American Journal of Plant Sciences, 3, 846-853.

https://doi.org/10.4236/ajps.2012.37102

[16] Soltani, N., Nurse, R.E., Shropshire, C. and Sikkema, P.H. (2013) Weed Control in White Bean with Pendimethalin Applied Preplant Followed by Postemergence Broadleaved Herbicides. The Open Plant Science Journal, 7, 24-30. https://doi.org/10.2174/1874294701307010024

[17] Soltani, N., Nurse, R.E., Van Eerd, L.L., Shropshire, C. and Sikkema, P.H. (2010) Weed Control, Environmental Impact and Profitability with Trifluralin plus Reduced Doses of Imazethapyr in Dry Bean. Crop Protection, 29, 364-368. https://doi.org/10.1016/j.cropro.2009.07.011 